\title{
IN VITRO KINEMATICS OF HUMAN NATIVE KNEES: A DATABASE OF 60 SPECIMENS
}

\author{
Luc Labey (1), Johan Bellemans (2), Yan Chevalier (1), Bilal El-Zayat (3), Susanne \\ Fuchs-Winkelmann (3), Petra Heesterbeek (4), Thomas Heyse (3), Jacek Kowalczewski \\ (5), Tomasz Okon (5), Silvia Pianigiani (6), Carsten Tibesku (7), Hilde Vandenneucker \\ (2), Jan Victor (8), Ate Wymenga (4), Bernardo Innocenti (1)
}

\author{
1. European Centre for Knee Research, Smith \& Nephew, Belgium; 2. U.Z. \\ Pellenberg, K.U. Leuven, Belgium; 3. University Hospital Marburg, Germany; 4. \\ University Hospital Nijmegen, The Netherlands; 5. Institute of Rheumatology, Poland; \\ 6. I.R.C.C.S. Istituto Ortopedico Galeazzi, Italy; 7. Sporthopaedicum straubing, \\ Germany; 8. AZ St-Lucas, Belgium
}

\section{Introduction}

Human knee kinematics has been widely studied, but data collected so far is fragmented. In vivo data is either based on three dimensional (3D) motion capture techniques or on fluoroscopy. 3D motion capture is not very accurate and shows only knee rotations. Fluoroscopy is more accurate but can not easily be applied to larger groups of healthy subjects. In vitro data has also been published, but only for small numbers of specimens.

The aim of this work is to present accurate morphology and tibio-femoral in vitro kinematics of 60 human knees using a single methodology.

\section{Methods}

Sixty fresh frozen full leg cadaver specimens were used. Frames with reflective markers were rigidly fixed to tibia and femur and a CT scan was made. Femur and tibia were embedded, properly aligned in frontal and sagittal planes. The medial and lateral hamstrings tendons were prepared for attachment to constant load springs (50 N each). The quadriceps tendon was prepared to be clamped to a motor. The knee rig provides six degrees of freedom to the knee joint. It can simulate loaded open chain and squatting motions. Infrared cameras continuously recorded the trajectories of the markers.

Each specimen was fixed in the rig and subjected to three motion patterns: a passive motion cycle, an open chain extension with a $3 \mathrm{~kg}$ weight hung to the distal tibia, and a squat between $30^{\circ}$ and $120^{\circ}$ of flexion with a constant ankle force of $130 \mathrm{~N}$.

Based on the CT, 3D models of tibia and femur were made and bony landmarks identified to determine coordinate frames for the bones. The marker trajectories were transformed to anatomical meaningful rotations and translations.

Tibial axial rotation and ab-adduction, and translations in antero-posterior and medio-lateral direction, were obtained as a function of flexion angle. The relative location and orientation of the optimal flexion axis with respect to anatomical landmarks was also determined.

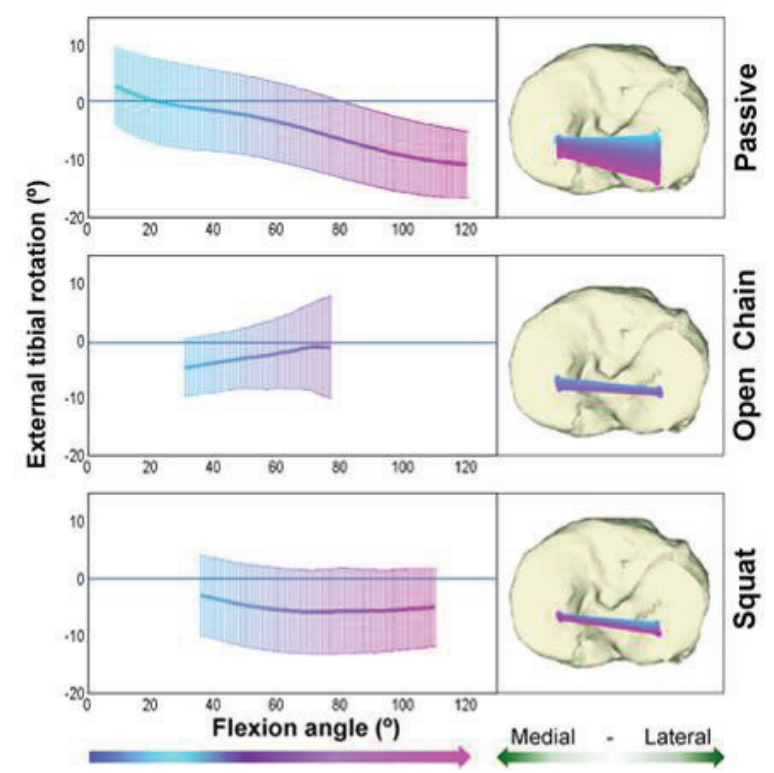

Figure 1: Mean tibio-femoral kinematics in passive, simulated open chain and squat motions.

\section{Results}

Rotations and translations depended on the loading regime (Figure 1). The tibia rotates internally with increasing flexion during passive motion by $20^{\circ}$ (standard deviation (SD): $4.8^{\circ}$ ). Minimal rotation was observed during squat $\left(3^{\circ}, \mathrm{SD}: 4.1^{\circ}\right)$. During open chain the tibia rotates externally with increasing flexion $\left(4^{\circ}, \mathrm{SD}: 7^{\circ}\right)$. The femoral medial condyle centre is more stable than the lateral during passive and squatting motions, but not during open chain. Variability among specimens was high, for all loading regimes.

\section{Discussion}

For the first time, motion data has been collected using the same methodology on a large collection of specimens for motor tasks which are functionally relevant. Knee kinematics was highly variable and was dependent on muscle and external loads within specimens and morphological differences between specimens. 